\title{
Blinking fluorophores: what do they tell us about protein dynamics?
}

\author{
C.R. Bagshaw ${ }^{1}$ and D. Cherny
}

Department of Biochemistry, University of Leicester, Leicester LE1 9HN, U.K.

\begin{abstract}
The ability to detect emission from a single fluorophore presents a powerful tool to probe the dynamic properties of protein molecules during their interactions with ligands. Here, different classes of experiments are reviewed and a distinction is drawn between experiments that monitor signals from a large number of proteins, one molecule at a time, from those that follow a single protein molecule over many individual cycles. The latter approach is potentially capable of resolving dynamic heterogeneity, such as that displayed by enzymes that fluctuate between high and low activity states. Other factors that need to be considered are the origin of the fluctuations in the emission signal and the extent to which this represents the properties of the protein under investigation, as opposed to the probe itself. Most fluorophores show fluctuations in their emission rates, termed flickering, blinking or intermittency, which may occur on a similar time-scale as the event under investigation.
\end{abstract}

\section{Introduction}

The detection of single catalytic turnovers of a single enzyme molecule using fluorescence methods was reported more than a decade ago $[1,2]$. The crucial advance that was necessary for single fluorophore detection was a reduction of the background signal by minimizing the observation volume (typically in the femtolitre range) using various forms of microscopy. The major limitations of these methods now come from the photophysical properties of fluorophores themselves. Even bright fluorophores only emit approx. $10^{6}$ photons before photobleaching and, of these, only approx. $10 \%$ may reach the detector. Although this number of photons is readily detected with little shot noise (proportional to the square root of the photon count), it becomes a major limitation when a reaction time course is monitored and the total count is divided over a number of time bins.

There are two basic approaches to single-molecule assays. Either the enzyme is fixed and is monitored over as many interaction cycles as possible or it is freely diffusing and many different molecules are assayed, one molecule at a time [3]. In principle, the former is better because an enzyme is observed as it goes though its full repertoire of its states. However, photodamage may limit the number of cycles that can be observed. When molecules are freely diffusing, their properties are recorded for the short time that they are present in the observation volume (typically up to a few milliseconds), but millions of molecules may be studied by

Key words: cyanine dye, fluorophore blinking, green fluorescent protein (GFP), photobleaching, protein dynamics, single-molecule enzymology.

Abbreviations used: FRET, Förster (fluorescence) resonance energy transfer; GFP, green fluorescent protein; PEG, poly(ethylene glycol); YFP, yellow fluorescent protein.

${ }^{1}$ To whom correspondence should be addressed (email crb5@le.ac.uk). building up the signal over many minutes. This constitutes a form of FCS (fluorescence COSY). Fluctuations in the fluorescence signal during the transit time of the molecule through the observation volume may correspond to steps in the catalytic cycle. In some respects, this assay is an ensemble method because the signal that is analysed comes from a large number of different molecules. However, by recording one molecule at a time, the technique has excellent dynamic range and can resolve intermediates present at low concentrations or with unique spectral properties that would be masked in conventional ensemble measurements.

\section{Single-molecule enzymology}

There are several ways of executing kinetic assays at the single-molecule level. An enzyme can be immobilized within a gel or on a glass/silica surface and the substrate tagged with a fluorescent probe. Provided the fluorescent substrate is kept at a low concentration (typically $<50 \mathrm{nM}$ ), fluorescence emission from the volume element containing the enzyme is indicative of the formation of an enzyme-ligand complex. The uncomplexed fluorescent substrate diffuses in and out of the volume element on a sub-millisecond time scale and does not build up a discrete image, but contributes to the background signal. The lifetime of the fluorescence spot provides a direct measure of the turnover time of the enzyme. However, the quantized decrease in fluorescence when the fluorescent product is released cannot be distinguished from photobleaching for any particular event. Nevertheless, the probability of photobleaching can be assessed by determining the dependence of the observed lifetime on the intensity of the excitation light. An elegant variation of this approach is to use a substrate combined with a fluorophore whose fluorescence 
is suppressed until catalysis occurs, at which point a single fluorogenic product molecule is detected [4]. These assays have revealed that many enzymes show fluctuations in the catalytic activity on the time scale of seconds to minutes [5].

For a basic enzyme-catalysed reaction of the type $\mathrm{E}+\mathrm{S} \leftrightarrow \mathrm{ES} \rightarrow \mathrm{E}+\mathrm{P}$ (where $\mathrm{E}$ is enzyme and $\mathrm{S}$ is substrate) at saturating $[\mathrm{S}]$, the mean time to produce a fluorescent product $\mathrm{P}$ is the reciprocal of the turnover rate $k_{\text {cat }}$. An ensemble single-turnover measurement (e.g. by stopped-flow methods) should reveal a single exponential decay of the ES complex with a half-time of $0.69 / k_{\text {cat }}$, which defines the time in which there is a $50 \%$ probability that a single ES complex will release product $P$. In the simple case, the lifetime of the ES complex varies stochastically and is exponentially distributed. Furthermore, there is no correlation between the lifetime of one event and the next. However, this is not always the case. Often an individual enzyme molecule shows phases of high and low activity and fluctuates between these phases on a time scale longer than individual catalytic events [5]. Ensemble single-turnover assays should reveal this heterogeneity through the deviation from a single exponential decay, but this may be experimentally difficult to do if the populations with the extremes in activity are less than $10 \%$ (dependent on the signal-to-noise ratio of the assay). If deviation from a single exponential is detected, stopped-flow studies would not provide information about whether the heterogeneity is static or dynamic. Thus singlemolecule enzyme assays can yield unique information in this regard.

\section{The relationship between protein function and folding}

The recent findings of fluctuations in enzyme activity lead to the conclusion that a rate constant is not constant, but rather it may exhibit large temporal fluctuations associated most probably with fluctuations of protein conformations [5]. These dynamics are related to the mechanism of protein folding. Many simple proteins appear to follow a two-state folding mechanism. However, the unfolded 'state' is not a single species but is near continuum of rapidly interconverting conformations. This leads to the concept of a folding funnel in which these conformers collapse into the favourable native state. But what is at the bottom of the funnel? Is there a just single native state? Most enzymes show some form of induced fit on interacting with substrates and many show quite substantial domain motions or quaternary rearrangements. This has led to the idea that the apo state can take on a number of conformations in the absence of any ligand and that substrate binding favours some states over others to 'cause' the conformational change [6]. The bottom of the folding funnel is therefore a rugged landscape in these instances, with a number of populated states. The two routes in which a conformational change occurs either before or after substrate binding are shown in the following scheme:

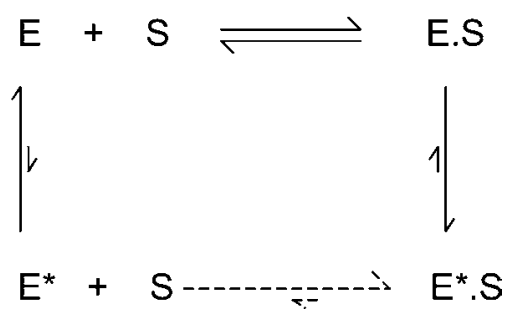

Although the $\mathrm{E}^{*}$ conformation binds $\mathrm{S}$ more tightly than $\mathrm{E}$, this route may be kinetically unfavourable. A closed enzyme active site (i.e. $\mathrm{E}^{*}$ ) may preclude the binding of substrate for steric reasons, even though the interacting side chains are in exactly the right place for favourable substrate interaction. In these circumstances, $\mathrm{E}^{*}$ would be an inhibitory state. If the $\mathrm{E} \leftrightarrow \mathrm{E}^{*}$ transition occurs on a longer time scale than subsequent catalytic events, then the catalytic activity would show fluctuations at the single-molecule level. This might be considered an unlikely scenario in this particular case because the $\mathrm{E} \leftrightarrow \mathrm{E}^{*}$ transition is likely to be at least as fast as the $\mathrm{ES} \leftrightarrow \mathrm{ES}^{*}$ transition, but other metastable states of $\mathrm{E}$ could contribute to slower transitions.

Reactions of the type $\mathrm{E} \leftrightarrow \mathrm{E}^{*}$ can be characterized by classical relaxation methods; however, if the equilibrium is strongly in favour of $\mathrm{E}$, then detection of $\mathrm{E}^{*}$ may be difficult. Single-molecule methods have the advantage of a better dynamic range [7]. A FRET [Förster (fluorescence) resonance energy transfer] pair located across the active site of an enzyme may report on the open to closed $\mathrm{E} \leftrightarrow \mathrm{E}^{*}$ transition, even if $\mathrm{E}^{*}$ is present for less than $1 \%$ of the time. Nonetheless, there remains the technical difficulty of knowing if the fluctuations in the FRET signal represent movement of the protein or properties of the fluorophores.

\section{Origins of fluorophore blinking}

Single-molecule enzyme assays using fluorescence detection attempt to relate a quantized event in the signal with a reaction step of the enzyme. However, in some instances, the events will represent the photophysical properties of the fluorophore rather than reflecting the behaviour of enzyme of interest. Emission can be terminated by photobleaching, but the probability of this can be determined from the dependence of termination on excitation intensity. In addition, the fluorophore may show spontaneous fluctuations in emission, which are more difficult to disentangle from genuine turnovers of the enzyme. These fluctuations are termed flickering (for an unresolved continuum) or blinking/intermittency (where discrete dark states exist for many milliseconds to hours). There are several causes of these fluctuations. Triplet-state formation is a contributing factor that can operate over a wide range of time scales. Polarization effects are also important at the single-molecule level, where slow rotations of the fluorophore (compared with the acquisition time bin) will give rise to changes in efficiency of excitation and emission detection. A more significant problem is spontaneous or 
Figure 1 | The origin of YFP blinking

(a) Single YFP molecules fused to a Dictyostelium myosin II motor domain and attached to an actin filament, viewed by TIR (total internal reflection) fluorescence microscopy. (b) Intensity profile of the arrowed spot showing binding of the YFP-myosin to actin followed by extensive blinking. (c) Autocorrelation analysis of a blinking YFP molecule, yielding a rate constant of $2.7 \mathrm{~s}^{-1}$. (d) Ensemble pH jump of YFP from pH 6.0 to a final pH of 7.3 measured using a stopped-flow instrument to yield a rate constant of $1.2 \mathrm{~s}^{-1}$. Blinking arises from the equilibrium, YFP- $+\mathrm{H}^{+} \leftrightarrow \mathrm{YFPH}$ and is activated by the intense excitation light used in fluorescence microscopy. For further details, see [11].

(a)

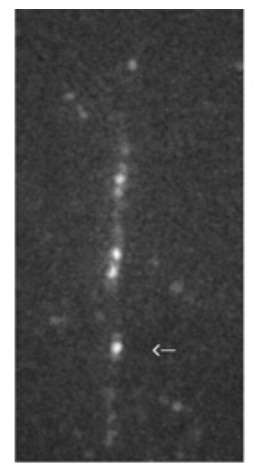

(c)

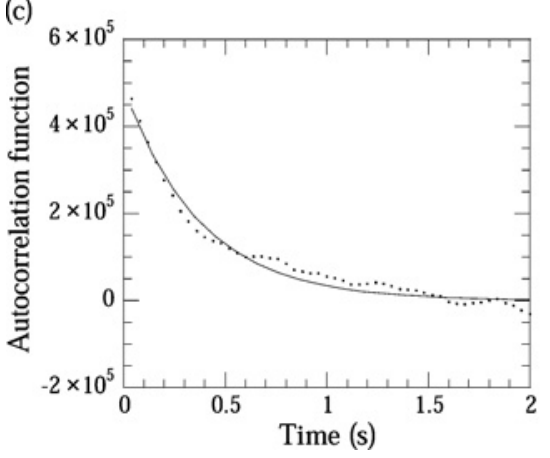

(b)

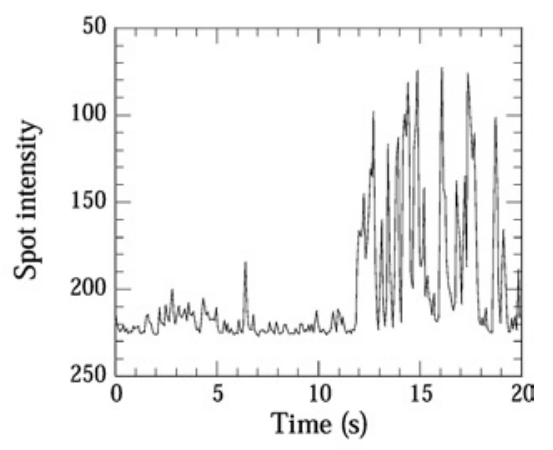

(d)

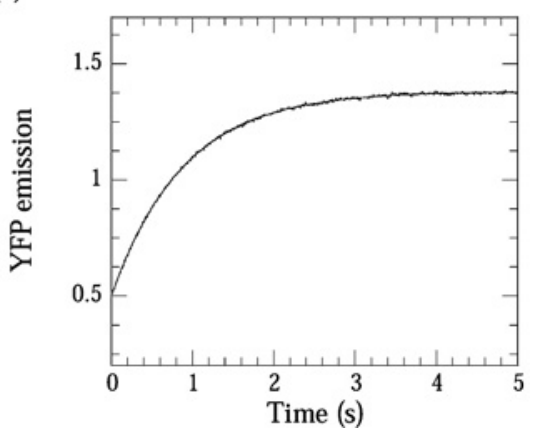

photo-induced isomerizations of the fluorophore. It is well known that excitation of conjugated organic dyes assists in photoisomerization because $\pi$-electrons in an anti-bonding orbital allow free rotation about the remaining sigma bond. If the absorption spectra of the cis and trans isomers differ, then excitation at a single wavelength can bias the population towards one isomer (the so-called photochromic effect). Thus one isomer can be trapped in a dark state for some time, if it does not absorb efficiently at the excitation wavelength because the ground-state barrier for the cis-trans isomerization is very high. If the recovery time is on the same time scale as enzyme turnover, this blinking may be difficult to distinguish from the genuine enzyme turnover. Furthermore, recently it was proposed that blinking need not be associated with intrinsic properties of the fluorophore but depends on the local environment $[8,9]$. We considered this possibility to account for the heterogeneous behaviour of Cy3-ATP in myosin ATPase assays, in which a subpopulation showed extensive blinking [10].

GFP (green fluorescent protein) and its variants are widely used as probes in cell biology. YFP (yellow fluorescent protein) shows spontaneous blinking in emission on the seconds time scale as a result of a proton-coupled isomeriz- ation (Figure 1). As the excitation intensity is increased, the blinking rate increases because photon absorption drives the protonation cycle [11]. GFP blinks on the millisecond time scale, in line with protonation studies at the ensemble level. However, when two GFP molecules dimerize, the low fluorescence protonated state becomes more favourable $[12,13]$ and blinking could become prevalent on a longer time scale. This phenomenon could also lead to difficulties in counting the number of GFP subunits in a single molecular complex from the initial intensity and number of photobleaching steps resolved.

The cyanine dye, Cy5, also shows extensive blinking. Cy5 blinking is markedly affected by the presence of oxygen and oxygen scavengers, indicating that triplet-states are a possible source of fluctuations. Radical cation and cis-trans isomerizations are other mechanisms that could contribute to blinking. Following Cy5 bleaching, illumination of the dark Cy5 isomer with green light restores the high fluorescence state $[14,15]$. This has interesting consequences for FRET studies when used as an acceptor with $\mathrm{Cy} 3$, for example. When in close proximity, Cy5 is an efficient acceptor of the energy of the excited $\mathrm{Cy} 3$ state and quenches $\mathrm{Cy} 3$ emission. When the excited Cy5 enters the dark state, the 
Figure 2 | Single-molecule FRET showing effect of Cy5 blinking

(a) A RNA-DNA 15-mer duplex was immobilized via a streptavidin-biotin link to the quartz slide covered with PEG [poly(ethylene glycol)]/PEGbiotin [18] as a model system in which Cy3 and (y5 were held approx. $5 \mathrm{~nm}$ apart. (b) Cy3 was excited at $532 \mathrm{~nm}$ and the Cy3 (grey line) and Cy5 FRET (black line, offset by +100 for clarity) emission was monitored simultaneously using dual-view optics and an electron-multiplier CCD (charge-coupled-device) camera. Events at 18 and $23 \mathrm{~s}$ correspond to cy5 blinking, so relieving the quenching of Cy3. At $26 \mathrm{~s}$, the Cy5 photobleaches (or goes into a long-lived dark state).

(a)

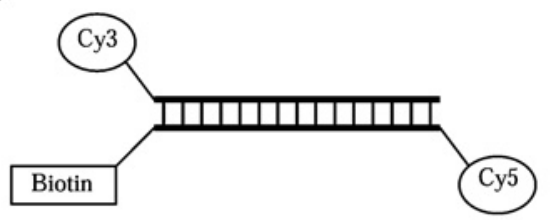

(b)

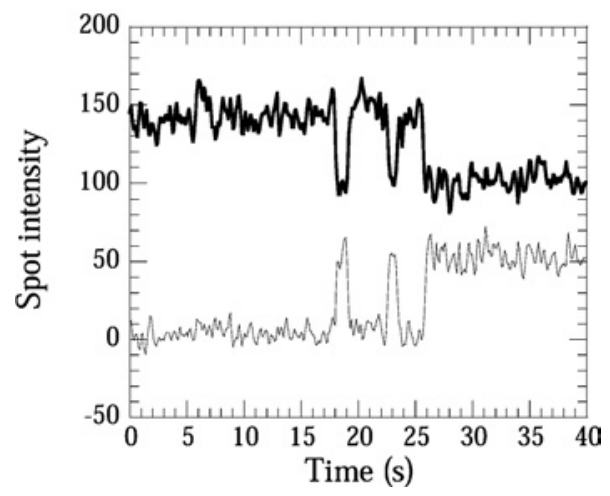

absorption spectrum is also changed and no longer quenches $\mathrm{Cy} 3$ emission. But the excitation light for $\mathrm{Cy} 3$ excites the Cy5 dark isomer and so it can quickly return to the starting fluorescent acceptor state. This behaviour gives anticorrelated blinking of the $\mathrm{Cy} 3$ and $\mathrm{Cy} 5$ emission until one component is irreversibly photobleached (Figure 2 and [16]). If this FRET pair is used to explore protein dynamics on the same time scale as blinking, then careful control experiments are required to establish the behaviour of the protein rather than that of the probe $[7,17]$. On the other hand, if the protein dynamics are much faster or much slower than blinking, then the latter provides a built-in calibration of the FRET signal, as the acceptor is temporarily removed and restored. There is another consequence of acceptor blinking. If the acceptor blinks rapidly and spends a significant amount of time in a dark (non-accepting) state during the time-binning of the data, the FRET efficiency may be underestimated, leading to an overestimation of inter-dye distance.

In summary, single-molecule studies have provided some unique information about enzyme activity. However, careful controls are required to establish the properties of the fluorescent probes themselves, before systems can be fully interpreted.

This work was supported by the Biotechnology and Biological Sciences Research Council, The Wellcome Trust and The Leverhulme Trust.

\section{References}

1 Funatsu, T., Harada, Y., Tokunaga, M., Saito, K. and Yanagida, T. (1995) Nature 374, 555-559

2 Lu, H.P., Xun, L. and Xie, X.S. (1998) Science 282, 1877-1882

3 Gell, C., Brockwell, D. and Smith, A. (2006) Handbook of Single Molecule Fluorescence Spectroscopy, Oxford University Press, Oxford

4 English, B.P., Min, W., van Oijen, A.M., Lee, K.T., Luo, G., Sun, H., Cherayil, B.J., Kou, S.C. and Xie, X.S. (2006) Nat. Chem. Biol. 2, 87-94

5 Min, W., English, B.P., Luo, G., Cherayil, B.J., Kou, S.C. and Xie, X.S. (2005) Acc. Chem. Res. 38, 923-931

6 Ma, B., Kumar, S., Tsai, C.J. and Nussinov, R. (1999) Protein Eng. 12 713-720

7 Weiss, S. (2000) Nat. Struct. Biol. 7, 724-729

8 Schuster, J., Cichos, F. and von Borczyskowski, C. (2005) Appl. Phys. Lett. 87, 051915

9 Yeow, E.K.L., Melnikov, S.M., Bell, T.D.M., De Schryver, F.C. and Hofkens, J. (2006) J. Phys. Chem. A 110, 1726-1734

10 Bagshaw, C.R. and Conibear, P.B. (2000) Single Mol. 1, 269-275

11 McAnaney, T.B., Zeng, W., Doe, C.F., Bhanji, N., Wakelin, S., Pearson, D.S., Abbyad, P., Shi, X., Boxer, S.G. and Bagshaw, C.R. (2005) Biochemistry 44, 5510-5524

12 Ward, W.W., Prentice, H.J., Roth, A.F., Cody, C.W. and Reeves, S.C. (1982) Photochem. Photobiol. 35, 803-808

13 Zeng, W., Seward, H.E., Malnasi-Csizmadia, A., Wakelin, S., Woolley, R.J., Cheema, G.S., Basran, J., Patel, T.R., Rowe, A.J. and Bagshaw, C.R. (2006) Biochemistry 45, 10482-10491

14 Bates, M., Blosser, T.R. and Zhuang, X.W. (2005) Phys. Rev. Lett. 94 , 108101

15 Heilemann, M., Margeat, E., Kasper, R., Sauer, M. and Tinnefeld, P. (2005) J. Am. Chem. Soc. 127, 3801-3806

16 Sabanayagam, C.R., Eid, J.S. and Meller, A. (2005) J. Chem. Phys. 123, 224708

17 Kozuka, J., Yokota, H., Arai, Y., Ishii, Y. and Yanagida, T. (2006) Nat. Chem. Biol. 2, 83-86

18 Ha, T., Rasnik, I., Cheng, W., Babcock, H.P., Gauss, G.H., Lohman, T.M. and Chu, S. (2002) Nature 419, 638-641

Received 19 July 2006 